\title{
Moderation of Preference Reversals in the Long Run
}

\author{
Douglas H. Wedell and Ulf Böckenholt \\ University of Illinois at Urbana-Champaign
}

\begin{abstract}
This study explored how preference reversals, induced by changes in response mode (choice vs. pricing), are moderated by how lotteries are represented, as being played 1, 10, or 100 times. Ss chose which of a pair of gambles they preferred to play and determined the minimum selling price of each gamble. Replicating previous research, the preference reversal was obtained for single-play representations: Ss tended to choose the gambles with the higher probabilities of winning, but priced them lower. However, for multiple-play representations, preference reversals were reduced, and consistency between pricing and choice behaviors was increased. Both response modes were sensitive to differences in the expected values of gambles, but sensitivity did not vary significantly with the number of plays. These results support the hypothesis that violations of expected utility theory are reduced for multiple-play gambles.
\end{abstract}

The evaluation of a monetary gamble requires consideration of two basic types of information: (a) the probabilities associated with winning and losing, and (b) the amounts to be won and lost. The well-documented phenomenon of preference reversals provides strong evidence that the processing of this information depends on how the evaluation is expressed (Goldstein \& Einhorn, 1987; Grether \& Plott, 1979; Lichtenstein \& Slovic, 1971, 1973; Lindman, 1971; Slovic \& Lichtenstein, 1983). When presented with two gambles close in expected value, many subjects choose a bet with a high probability of winning a modest amount of money (P-bet) over a bet with a low probability of winning a large amount (\$-bet), even though they place a higher minimum selling price on the \$-bet. When confronted with the apparent inconsistency in their behavior and given the chance to change either of their responses, the majority of these subjects stand by their decisions (Slovic, 1986).

In the typical preference reversal task, response modes may vary in two ways: (a) subjects either make a choice between two lotteries or make a judgment of each separately, and (b) the focus is either on the monetary value of the gamble or on its attractiveness. Goldstein and Einhorn (1987) manipulated these two factors independently and demonstrated preference reversals associated with each. Although recent research (Tversky, Sattath, \& Slovic, 1988) has demonstrated preference reversals for nonprobabilistic stimuli, the vast majority of preference reversal studies have used gambles as stimuli. In this article, we explore the possibility that these preference reversals are largely due to the way in which people evaluate probabilities for unique, one-shot events. Two experiments test this hypothesis by varying the number of times a gamble

We would like to acknowledge the helpful comments of Jerome Busemeyer, Lola Lopes, and Elke Weber on an earlier version of this article. This research was carried out while Douglas $\mathrm{H}$. Wedell was at the University of Illinois on postdoctoral traineeship, with ADAMHA National Support Award MH142557 (Lawrence E. Jones, Training Director).

Correspondence concerning this article should be addressed to Douglas H. Wedell, Department of Psychology, University of South Carolina, Columbia, South Carolina 29208. is represented as being played, from a single play to 100 plays. When presented with gambles that are played multiple times, subjects may tend to consider them in terms of their expected, long-run outcomes under both pricing and choice conditions and hence exhibit greater consistency across response modes.

\section{Decision Making in the Long and Short Run}

Expected utility theory is based on the premise that a longrun perspective should be applied when considering single instantiations of probabilistic events (Von Neuman \& Morgenstern, 1947). Thus, one should simply choose the alternative that has the expectation of greatest utility in the long run. However, there is a growing body of evidence to suggest that people do not naturally apply a long-run perspective to shortrun probabilistic events. In an influential article, economist Paul Samuelson (1963) related an anecdote that brings home this point. At a luncheon he asked his colleagues if they would be willing to stake $\$ 100$ for a $50 / 50$ chance to win $\$ 200$, which can be denoted as $(-\$ 100, .50, \$ 200)$. The article centers on the reply of one distinguished colleague who refused the bet unless he was guaranteed a chance to play 100 times, his reason being that in the long run he was "virtually sure to come out ahead." Samuelson suggested that such a preference pattern was irrational and violated expected utility theory (for a formal proof, see Tversky \& Bar-Hillel, 1983).

Lopes (1981), however, questioned the application of the long-run perspective to short-run events. For example, in a computer simulation she demonstrated the profitability of selling a bet with infinite expected value (as described in the St. Petersberg paradox) for only $\$ 100$. Although in the very, very long run such an enterprise would be doomed to bankruptcy, at the end of her computer simulation of 1 million trials for each hypothetical business, 90 out of 100 businesses were still in the black (and making considerable profit).

In a similar way, we can imagine Samuelson's colleague simulating possible outcomes for playing the proposed gamble just once or 100 times. Playing the bet once, it is clear that half of the time one loses. Playing the bet 100 times, the probability of coming out a loser is less than 1 in 2,000. These odds were apparently more preferable to Samuelson's col- 
league, even though he ran the possible risk of losing much more (however, the probability of losing $\$ 1,000$ or more is less than 1 in 25,000).

These examples suggest a wide gap between normative prescriptions and actual behavior. One aim of this article is to determine whether this gap is narrowed when probabilistic events are represented as multiple instantiations. There is mounting evidence that choices among probabilistic alternatives differ depending on whether alternatives are represented as being instantiated once or multiple times (Coombs \& Bowen, 1971; Coombs \& Meyer, 1969; Keren \& Wagenaar, 1987; Montgomery \& Adelbratt, 1982). For example, Keren and Wagenaar (1987) demonstrated that certainty and possibility effects are eliminated when gambles are represented as being played repeatedly (10 or 100 times). Because these effects constitute violations of expected utility theory (Kahneman \& Tversky, 1979), one interpretation of this finding is that multiple instantiations of gambles induce subjects to integrate probabilities with outcomes using a long-run perspective, bringing choice behavior more in line with expected utility theory. This interpretation implies that other violations of expected utility theory, such as the preference reversal phenomenon, may be moderated or eliminated by representing probabilistic alternatives as repeated instantiations.

\section{Consequences of Repeating Gambles for Preference Reversals}

Consider the following two bets: a P-bet in which one has a .9 chance to win $\$ 10$ and a .1 chance to win nothing (.9, $\$ 10$ ) and a $\$$-bet in which one has a .1 chance to win $\$ 100$ and a .9 chance to win nothing $(.1, \$ 100)$. In accordance with the typically observed preference reversal pattern (Slovic \& Lichtenstein, 1983), the majority of subjects would be expected to choose the P-bet over the \$-bet but to set a higher minimum selling price on the latter. Tversky et al. (1988) recently demonstrated that this pattern of results can be parsimoniously described by a weighted multiplicative model in which the exponential weight of the probabilities is low under the pricing condition and high under the choice or attractiveness judgment conditions. This model is consistent with basic anchoring and adjustment explanations of preference reversals, according to which pricing is anchored on the monetary outcomes with insufficient adjustment toward probabilities, and vice versa for choice. This type of descriptive explanation, however, does not address the potential effects of varying the number of times the gambles may be played on preference reversals. To determine this, one must specify these effects on both response modes, choice and pricing.

\section{Choice Behavior}

The selection of the P-bet over the \$-bet despite the higher expected value of the latter (\$9 vs. $\$ 10$ in the stated example) is consistent with the typical finding of risk aversiveness in the domain of gains (Arrow, 1971; Kahneman \& Tversky, 1979; Pratt, 1964). It is also consistent with the behavior of Samuelson's colleague, who refused to play the single gamble despite a fairly high expected value of $\$ 50$. Although one way to model this behavior is in terms of a negatively accelerated (concave) utility function, an alternative interpretation focuses on the concept of aspiration level.

Consider that although Samuelson's colleague was unwilling to play the single gamble, he found the repeated gamble quite attractive, because he felt he had virtually no chance of losing. This pattern is consistent with the idea that in choice situations, individuals compare each alternative to current needs and expectations as summarized by an aspiration level (Lopes, 1983; Payne, Laughhunn, \& Crum, 1980; Schneider \& Lopes, 1986; Siegel, 1957; Simon, 1955). If the aspiration level were set at the status quo, then Samuelson's single gamble would seem unacceptable, because there is a high probability (.5) of falling below the aspiration level; however, the multiple-play gamble would seem attractive, because nearly all possible outcomes fall well above the aspiration level.

More generally, choice among risky prospects can be conceived as a two-stage process (Kunreuther \& Wright, 1979; Lopes, 1984) in which (a) alternatives with unacceptably low probabilities of exceeding the aspiration level are first eliminated from the choice set and (b) an alternative is selected from the remaining set on the basis of some other criterion, such as maximizing expected value or potential gain. The first stage of this process is consistent with the prominence hypothesis proposed by Tversky et al. (1988), which states that choice behavior follows a more lexicographic strategy, the more prominent dimension receiving greater weight. However, the two-stage aspiration level model provides an explanation of why probabilities are more prominent in choice among gambles. Furthermore, it can be used to predict the effects of increasing the number of plays. To explain why the P-bet is chosen over the $\$$-bet in the single-play situation, the model must assume that the aspiration level is greater than zero but less than the positive outcome value for the P-bet. In this way, the \$-bet tends to be eliminated during the first stage of the process because it has a low probability of exceeding the aspiration level, and so the P-bet is chosen.

Now consider a choice between playing the P-bet or \$-bet in our example 100 times. The $\$$-bet, even with its relatively low probability of winning, is virtually assured to yield a positive outcome in 100 plays. In general, as the number of plays increases, it becomes less likely that the $\$$-bet will be eliminated during the first stage of the choice process, and hence the relative preference for the P-bet should be reduced. Moreover, under these circumstances, the $\$$-bet may actually be preferred to the P-bet on the basis of its higher expected value or its possibility of greater gains. Thus, an aspirationlevel analysis implies that choice preferences should shift from the P-bet to the \$-bet as the number of plays increases, which is in the direction of the preference pattern exhibited in pricing.

\section{Pricing Behavior}

Given that choice preferences shift in favor of the \$-bet with multiple plays as described in the previous paragraph, a reduction of preference reversals will depend on how pricing 
behavior changes. For instance, if preferences as expressed in pricing show a similar shift in favor of the \$-bet, no reduction would occur. On the other hand, if the pricing preferences remained the same or shifted toward the P-bet with more plays, preference reversals would be reduced and perhaps eliminated. An aspiriation-level explanation of why pricing preferences would shift toward the P-bet is described in the General Discussion.

\section{Experiment 1: Single Versus Multiple Plays for High- and Low-Value Gambles}

The major purpose of Experiment 1 was to determine whether representing gambles as repeated events would reduce preference reversals. Gambles were represented as being played either 1 time or 10 times. The monetary values of the outcomes were also manipulated at two levels by doubling or halving payoffs from two-outcome gambles used in previous research (Goldstein \& Einhorn, 1987) to create high and low outcomes, respectively. The aspiration-level interpretation implies that manipulation of outcome level could in itself moderate preference reversals. If the outcomes for the P-bet all fall below the aspiration level, the \$-bet may be chosen even though it has a lower probability of winning. For example, a .01 chance to win $\$ 9$ may be preferred to a .99 chance to win $10 \uparrow$, if $10 \uparrow$ is viewed as a trivial gain. Given that pricing behavior remained unchanged, preference reversals should be reduced for the low outcome gambles. However, this prediction would not necessarily hold for multiple-play gambles because the low outcomes, when multiplied by the number of plays, might far exceed the aspiration level.

A second reason for manipulating outcome level is to partially unconfound the fact that as the number of plays increases, potential outcome levels increase as well. Thus, a reduction in preference reversals with more plays might be due to how subjects evaluate the higher potential outcomes rather than due to a change toward adopting a long-run perspective. For example, subjects might first multiply the outcomes by the number of plays and then evaluate the bets on the basis of the stated probability to win the new (increased) outcome level. However, for this type of process to lead to greater choice of the \$-bet, the underlying utility function would have to be convex (positively accelerated) instead of concave, which is usually postulated (Arrow, 1971). Furthermore, the proposed process would predict that choice of the \$-bet over the P-bet would increase with outcome value, which is just the opposite of the prediction from the aspiration-level interpretation.

\section{Method}

Design and subjects. The experimental design consisted of the factorial combination of four within-subjects variables-type of bet (P-bet or \$-bet), response mode (choice or pricing), number of plays ( 1 or 10), and value (high or low)-and one between-subjects variable, order of tasks (pricing first or choice first). Booklets consisting of eight experimental pages were constructed by the factorial combination of the within-subjects variables. Type of bet was the only variable that varied within a page, with each page consisting of four pairs of bets, a P-bet and a \$-bet making up a pair. Half of the subjects were randomly assigned to indicate prices first, the other half to indicate choices first. Within choice and pricing conditions, the order of the four different pages was randomized to counterbalance order effects. To minimize effects due to remembering responses to specific bets, the ordering of the bets within each pair was randomized, as was the position of the bets on each page. The basic dependent variable was the bet from each pair that was chosen or priced higher. Altogether, 55 University of Illinois undergraduates served as subjects to fulfill a course requirement.

Booklets. General instructions, printed on the first page of the experimental booklets, stated that the experiment concerned how people make decisions about bets. Subjects were told that none of the bets actually involved losing any money, and the following bet was given as an example: $50 \%$ chance to win $\$ 5.00$ and $50 \%$ chance to win $\$ 0.00$ (this is the exact phrase presented to subjects). Instructions described the two types of responses, choice and pricing. When choosing between bets, subjects were asked to imagine that they were given the chance to play one bet or the other. When pricing bets, subjects were told to determine the smallest price for which they would be willing to sell the bet and were admonished that the selected price should be sufficiently attractive for both the subject and potential buyers of the bets. Subjects were told not to feel that they had to use any type of complex computations but that they could simply follow whatever strategies they felt were appropriate to the task.

Specific instructions were printed on each of the remaining eight pages of the booklet, and below them were printed four pairs of bets. Each bet was enclosed in a rectangle, and its wording paralleled that of the example. Eight pairs of bets were used, each pair consisting of a P-bet (high probability to win a small amount) and a \$-bet (low probability to win a large amount). The pairs were constructed from four pairs used in previous research on preference reversals (Goldstein \& Einhorn, 1987): $(.97, \$ 4)$ versus $(.31, \$ 16) ;(.81, \$ 2)$ versus $(.19$, $\$ 9) ;(.94, \$ 3)$ versus $(.50, \$ 6.50)$; and $(.94, \$ 2.50)$ versus $(.39, \$ 8.50)$. The pairs of bets listed here were not used directly; instead, a set of four low-value bet pairs were constructed by halving outcome values, and a set of four high-value bet pairs were constructed by doubling outcome values.

P-bets appeared twice in the left column and twice in the right column on each page. In the 10-play condition, the phrase TEN TIMES TO PLAY was printed above each bet. Bets within each pair were placed close together for pages on which subjects were to indicate choices (by circling one bet from each pair). Choice instructions were as follows:

Below are listed four pairs of bets. You can think of each bet as representing a lottery ticket with the odds of winning and the amount to be won as described by the bet. For each pair of bets, imagine you can select 1 ticket $[10$ tickets] from one bet or the other. Thus you will play a bet just once [10 times]. For each pair, circle the bet you would prefer to play 1 time [10 times].

For pages on which subjects were to indicate prices, the bets within a pair were spaced more widely, and a blank line with a dollar sign was printed to the left of the bet. Pricing instructions were as follows:

Below are listed eight bets. You can think of each bet as representing a lottery ticket with the odds of winning and the amount to be won as described by the bet. Imagine that the lottery tickets are sold singly [in blocks of 10]. Your task is to determine the minimum selling price for each ticket [block of 10 tickets]. Indicate this price at the blank to the left of each bet.

Procedure. Subjects were tested either in groups of 8 to 12 or as part of a classroom exercise. They were told to read the instructions 


\section{LOW VALUE}
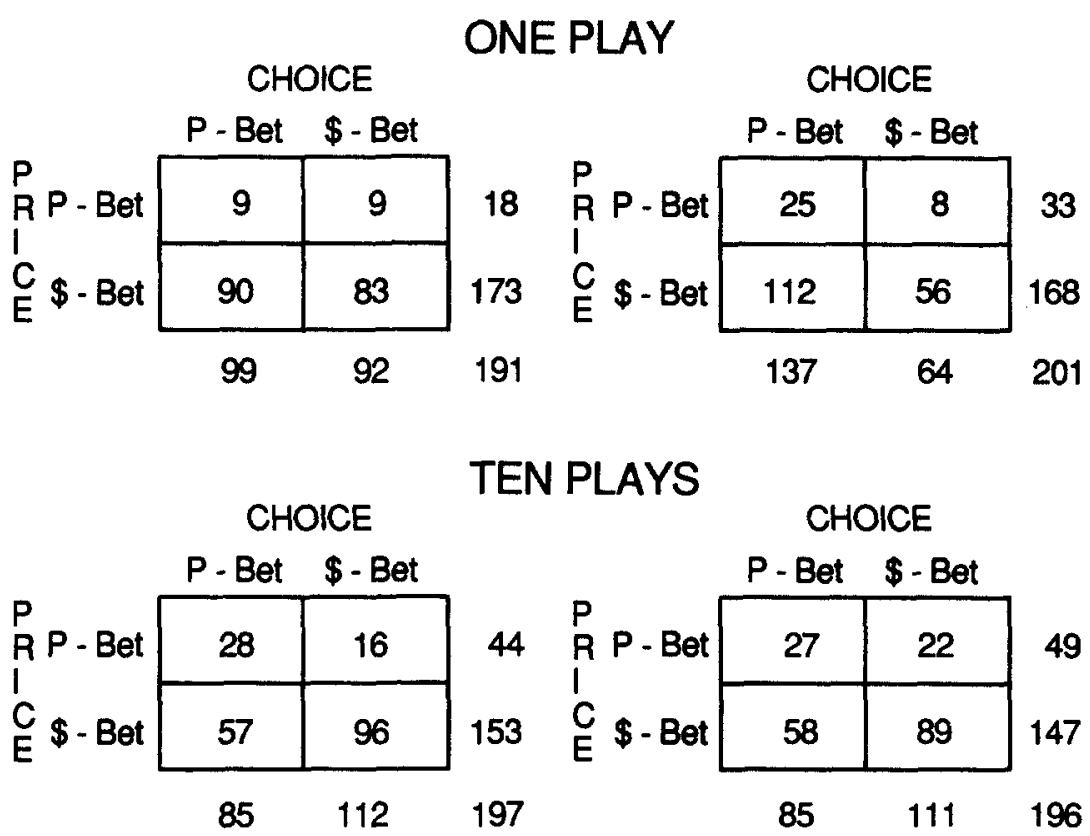

Figure 1. Contingency tables for data from Experiment 1. (Each subject is represented up to four times in each table. Data are not included for bet pairs in which the same price was assigned to P-bet and \$-bet.)

carefully as they worked their way through the booklets, from front to back. There was no time limit, although subjects typically finished within $15 \mathrm{~min}$.

\section{Results and Discussion}

Preference reversals. Figure 1 presents the results in contingency table form. The number of responses favoring P-bets and \$-bets under each response mode condition is segregated by the number of plays and the value of bets. For each $2 \times 2$ matrix, the number of inconsistencies is given by the offdiagonal. The number of preference reversals in the predicted direction is found in the bottom left cell. Typically, this value is compared to the number in the top right cell, which is viewed as representing baseline (chance) inconsistencies. The asymmetry in the type of inconsistencies observed is reflected by the marginal preferences: The predicted preference reversal is described by a greater marginal preference for the P-bet when choosing between bets than when pricing bets. A repeated-measures multivariate analysis of variance (MANOVA) was used to determine the effects of the experimental manipulations on preference reversals, the dependent variable being the number of times the P-bet was chosen over or priced over the corresponding $\$$-bet (coded as $1=\mathrm{P}$-bet $>\$$-bet, $-1=\$$ bet $>$ P-bet, and 0 for ties in the pricing condition).

An initial MANOVA that compared the pattern of results for the two basic orders (pricing first or choice first) revealed no significant main effect of order or interactions with the order variable; hence all analyses reported here combine the two groups. The significant main effect of response mode, $F(1$, $54)=54.62, p<.001$, reflected the overall expected preference reversal effect; however, preference reversals were significantly reduced for the repeated-play condition as evidenced by the Response Mode $\times$ Number of Plays interaction, $F(1,54)=$ $29.41, p<.001$. Planned comparisons revealed significant $(p<.05)$ response mode effects for both the single-play and the 10-play conditions. Thus, although increasing the number of plays significantly decreased the number of preference reversals, it did not eliminate them completely.

The decrease in preference reversals was fairly general across subjects. Only 1 of the 55 subjects showed no preference reversals in either the 1- or 10-play conditions. Of the remaining 54 subjects, $63 \%$ exhibited fewer preference reversals with more plays, $15 \%$ exhibited more preference reversals with more plays, and $22 \%$ had equal numbers of preference reversals for the 1 - and 10 -play conditions. ${ }^{2}$

\footnotetext{
${ }^{1}$ The marginal preferences shown in the contingency tables differed slightly from those on which the MANOVA was conducted because of the difference in how ties were coded. For the contingency tables, a tie on prices resulted in the elimination of the bet pair from the table, but for the MANOVA a tie on prices resulted in a coding of 0 , halfway between preference for P-bet (1) and preference for \$-bet $(-1)$. Although this difference in coding could potentially lead to fairly large differences in marginal preferences, this was not the case. The mean of absolute differences between marginal proportions was less than 0.01 for both Experiments 1 and 2, the largest deviation being 0.026 .

${ }^{2}$ An examination of the pricing responses suggested that several subjects did not take into account the manipulation of the number of plays; that is, their prices for the unique bets were the same as those for bets played 10 times. These subjects may have misunderstood instructions and priced each play instead of the set of plays, or
} 
Of particular relevance to understanding how the repeated gambles are processed is the determination of whether marginal preferences shifted for both response modes or only one mode (the observed interaction could result from either). To answer this question, we carried out planned comparisons (at $p<.05$ ) at each level of response mode. These tests revealed that choice preferences shifted significantly toward the \$-bet and price preferences shifted significantly toward the P-bet.

Increasing the value of the payoffs resulted in a greater overall preference for the P-bet, $F(1,54)=7.81, p<.01$; however, the effect of the value manipulation occurred only for the single-play bets as revealed by the Value $\times$ Plays interaction, $F(1,54)==7.23, p<.05$. The three-way Value $\times$ Response Mode $\times$ Number of Plays interaction failed to achieve significance, $F(1,54)=3.32, p>.05$. Planned comparisons (at $p<.05$ ) showed that choice preference for the P-bet over the \$-bet was greater for high-value bets in the 1play condition but did not differ significantly across value levels in the 10-play condition. This pattern of results is inconsistent with an interpretation that the observed reduction in preference reversals is due to a corresponding increase in outcome values for multiple-play bets. If this were the case, preference reversals should show a similar decrease with increase in outcome values. However, this pattern is consistent with the aspiration-level framework as well as with a concave utility function.

Consistency across response modes. It is important to note that differences in marginal preferences can be reduced without a corresponding increase in overall consistency. That is, the number of entries in the off-diagonal cells may remain the same but become more symmetric. To evaluate whether subjects became more consistent in their pricing-choice behaviors, we ran a two-way within-subjects MANOVA in which the dependent variable was the number of inconsistencies for each subject per condition (i.e., the sum of each subject's offdiagonal entries). Increasing the number of plays led to a significant increase in consistency between modes, $F(1,54)=$ $10.88, p<.01$. Thus, these results support the notion that with repeated plays, subjects adopt similar strategies in their pricing and choices. Consistency was also significantly higher for the low-values bets, $F(1,54)=4.06, p<.05$. This is in line with the interpretation that the P-bet failed to exceed subjects' aspiration levels, especially in the single-play condition, and thus led more subjects both to choose the \$-bet and to price it higher.

In summary, Experiment 1 demonstrates that preference reversals are reduced by allowing subjects multiple opportunities to play the same gamble. Both pricing and choice behaviors are affected, but in opposite directions, producing greater consistency of preferences across response modes.

they may simply have ignored the manipulation altogether. To investigate this possibility, we ordered subjects by difference in price between the 1- and 10-play conditions and segregated them into two groups on the basis of a median split. This between-subjects factor was included in a MANOVA; however, neither the main effect nor any interaction with this selected factor approached significance. Therefore, the results appear to hold even for those subjects whose overt pricing behavior was relatively insensitive to number of plays.

\section{Experiment 2: Sensitivity to Expected Value With Multiple Plays}

A major purpose of Experiment 2 was to investigate whether subjects are more sensitive to the expected value of a bet with repeated plays. In previous research (Montgomery $\&$ Adelbratt, 1982), subjects were asked how many opportunities to play a bet would induce them to make choices on the basis of expected value. The median number of plays suggested by subjects was 10 , implying that with repeated opportunities to play a bet, there should be greater sensitivity to expected value. However, because subjects in that experiment had been thoroughly indoctrinated with the theory of expected value and did not actually make choices, it seems important to directly test the hypothesis that the choice behavior of naive subjects will reflect greater sensitivity to expected value with multiple plays. Experiment 2 tested this hypothesis by manipulating the expected values of the bets so that for half of the pairs of bets the \$-bet had the higher expected value (as in Experiment 1) and for the other half the P-bet had the higher expected value. If representing gambles as multiple instantiations induces subjects to base their choice or pricing behavior more on the expected values of the bets, there should be a greater tendency to choose or price higher the bet with the higher expected value as the number of plays increases.

A second purpose of Experiment 2 was to explore what constitutes the long run. Although preference reversals were reduced in Experiment 1 by extending the number of plays from 1 to 10 , they were not eliminated altogether. Perhaps this is simply because 10 plays are not enough to consistently evoke a long-run perspective. Experiment 2 investigated this idea by extending the number of plays to 100 .

A third goal of Experiment 2 was to get some assessment of how subjects viewed the differences between single- and multiple-play situations. We did this by adding a short verbal questionnaire to the end of the booklets that asked subjects to describe their choice and pricing strategies and that also asked them whether and how these strategies were affected by increasing the number of plays for a bet.

\section{Method}

Design and subjects. Each page of bets represented the factorial combination of Type of Bet (P-bet or \$-bet) $\times$ Expected Value (P-bet higher or \$-bet higher). Six such pages were constructed by the further factorial combination of Response Mode (pricing or choice) $\times$ Number of Plays $(1,10$, or 100 ). Approximately half of the subjects were assigned to price bets first, the other half to choose between pairs first. Within choice and pricing conditions, the order of the three different pages was randomized to counterbalance order effects. As in Experiment 1, the ordering of the bets within each pair was randomized, as was the position of the bets on each page. Once again, the dependent variable was the bet from each pair that was chosen or priced higher. Subjects were 90 undergraduates sampled from the same population as in Experiment 1.

Bets. Four pairs of bets were used in Experiment 2: two in which the expected value of the P-bet was higher than that of the \$-bet, $(.97$ $\$ 8)$ versus $(.39, \$ 17)$ and $(.94, \$ 10)$ versus $(.19, \$ 42)$; and two in which the expected value of the $\$$-bet was higher, $(.94, \$ 7)$ versus 
$(.39, \$ 20)$ and $(.94, \$ 9)$ versus $(.31, \$ 32)$. As in Experiment 1 , a P-bet was always paired with a \$-bet.

Instructions. In Experiment 2, we altered the instructions slightly to further emphasize the manipulation of number of plays. The first page of the booklet once again consisted of general instructions that were essentially the same as those used in Experiment 1 . The main instructional differences were in the specific instructions presented on each page of the booklets. These attempted to make the manipulation of the number of repetitions more concrete and salient by repeatedly emphasizing the number of plays and by including an example of how the specific number of plays being considered altered the amount to be won.

\section{Results and Discussion}

Preference reversals. Figure 2 presents the results in contingency table form segregated by the number of plays and the expected value. As in Experiment 1, a MANOVA was used to analyze the effects of the manipulations on preference reversals with the dependent variable again being each sub- ject's marginal preferences for the P-bet. The main effect of response mode was significant, $F(1,89)=27.55, p<.001$, reflecting an overall preference reversal. There was, however, a significant Response Mode $\times$ Number of Plays interaction, $F(2,88)=8.51, p<.01$, indicating, as in Experiment 1, that preference reversals were reduced under repeated-play conditions. Planned comparisons (at $p<.05$ ) of the effects of response mode at each level of the number of plays indicated the preference reversal was statistically significant at the 1and 10-play levels but not at the 100-play level: Thus, the strong preference reversals that occurred for single-play bets were effectively eliminated by representing the bets as being played 100 times.

Unlike Experiment 1, a fairly large proportion of subjects (20 out of 90 ) showed no preference reversals for any of the conditions $(1,10$, or 100 plays). However, similar to Experiment $1,60 \%$ of the remaining 70 subjects exhibited fewer preference reversals for 100 plays than for 1 play, $23 \%$ exhibited the opposite trend, and $17 \%$ had equal numbers of reversals for both conditions. Thus, the results generalize
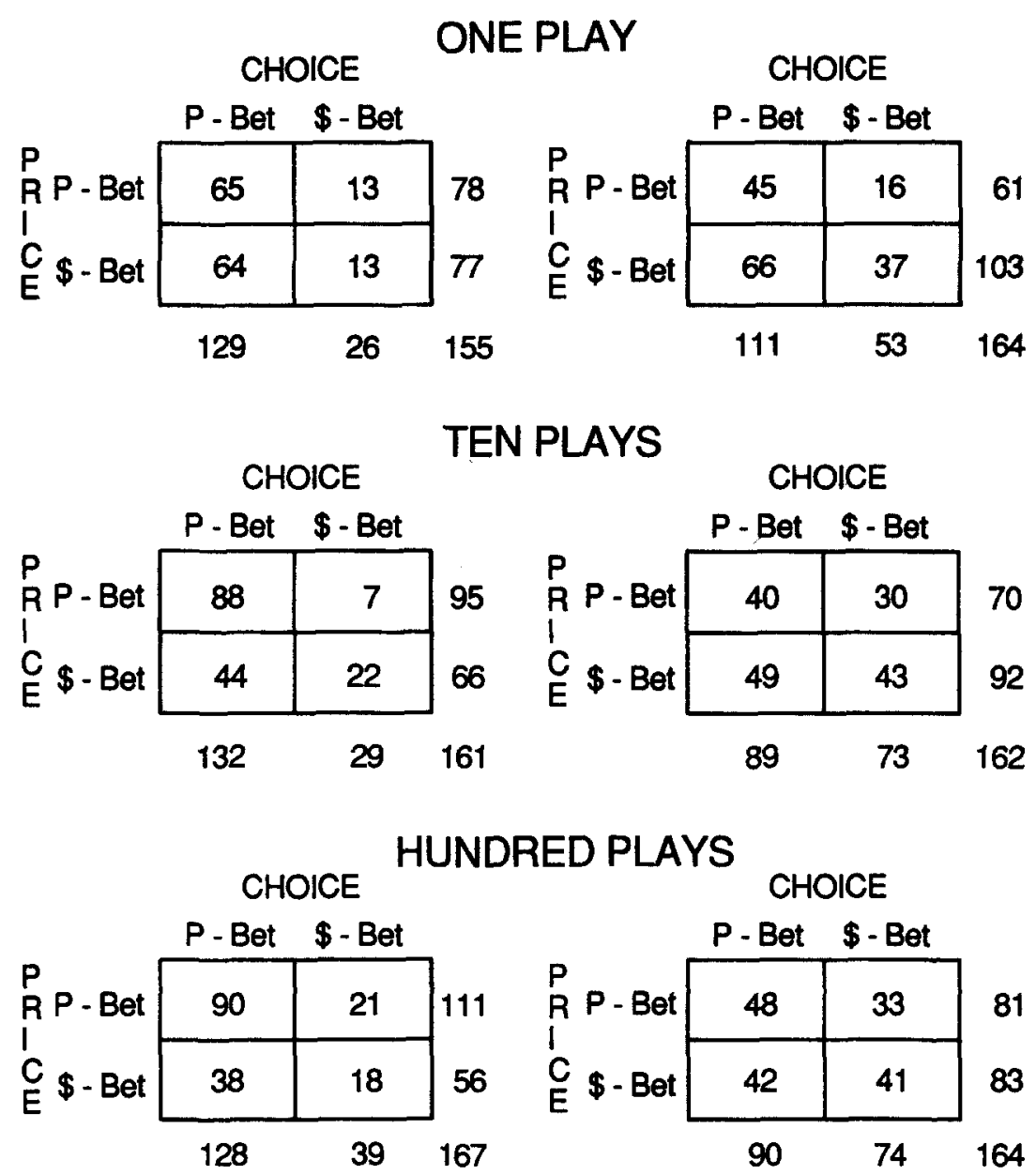

Figure 2. Contingency tables for data from Experiment 2. (Each subject is represented up to two times in each table.) 
across the majority of subjects who showed any inconsistencies at all.

A major focus of Experiment 2 concerned whether expected value might receive greater consideration under repeated-play conditions. On the whole, the subjects' choice and pricing behaviors were quite sensitive to the expected value, $F(1,89)$ $=69.72, p<.001$. A planned comparison revealed that this result holds for both subjects' choice and pricing behaviors $(p<.05)$. Although sensitivity appeared greater for multipleplay conditions, the Expected Value $\times$ Number of Plays interaction did not achieve significance, $F(2,88)=3.00, p>$ .05 . Planned comparisons showed that sensitivity to expected value increased significantly only from 1 to 10 plays. Thus, the results provide weak evidence that increasing the number of plays makes the expected value of the bets more salient or attractive.

Consistency across response modes. A MANOVA using the number of inconsistencies for each subject as the dependent variable revealed that subjects were significantly more consistent when the P-bet had the higher expected value, $F(1,89)$ $=4.45, p<.05$. This may, in part, reflect the tendency of the majority of subjects to choose the P-bet regardless of its expected value in relation to the \$-bet (the marginal preference for the P-bet never dropped below 50\%). Because the majority of preferences in the pricing mode favored whichever bet had the higher expected value, there was a greater tendency for choice and pricing preferences to overlap when the P-bet had the higher expected value. This difference in the marginal preferences between the two modes is consistent with the tendency for strong preference reversals when the \$-bet had the higher expected value. Although the number of plays had a strong effect on consistency between response modes in Experiment 1, consistency did not significantly increase with the repetition of bets in Experiment 2, $F(2,88)=2.59, p>$ .05 . The three-way interaction was also not significant $(p>$ .25).

Verbal reports. Four questions were printed on the last page of the experimental booklets. First, subjects were asked to describe how they chose between bets or priced bets. Table 1 classifies their responses into one of three categories on the basis of whether they claimed that they paid attention only to probabilities, only to prices, or to both prices and probabilities. A fourth category was formed for those subjects who could not be classified. Subjects' responses differed when describing their choice and pricing behaviors. For choice, the modal response was to choose the bet with the higher probability of winning. On the other hand, descriptions of pricing generally included taking both probabilities and amounts into account. Consistent with previous research (Slovic \& Lichtenstein, 1968), subjects were less able to verbalize their pricing behavior, with many of the unclassifiable statements voicing this problem explicitly (e.g., "I just guessed").

Subjects were also asked whether increasing the number of plays affected their choices or pricing. Table 2 shows the number of subjects responding yes or no and classifies explanations of the yes responses into three categories on the basis of whether they indicated that the likelihood of winning changed, the amount to be won changed, or some combination of the two changed. A fourth category was formed for those subjects who could be classified. Results were parallel for the two response modes. The majority of subjects declared that the number of times a bet was played did make a difference, with the modal explanation of the difference being that the "chance of winning" increased. Thus, the verbal protocols provide additional evidence that individuals consider probabilistic information differently for unique versus repeated events.

\section{General Discussion}

\section{Preference Reversal Phenomenon}

The results of Experiments 1 and 2 demonstrated that increasing the number of perceived opportunities to play gambles produces strong but opposing effects on pricing and choice behaviors. As the number of plays increases, preference for the P-bet over the \$-bet decreases for choice but increases for pricing. These opposing effects result in a reduction of response-mode induced preference reversals. In their review of the preference reversal literature, Slovic and Lichtenstein (1983) pointed out the general failure of researchers to eliminate reversals by manipulation of various task and contextual factors. A major empirical contribution of the present set of experiments is the documentation of a task variable - number of plays-that greatly reduces reversals.

One possible response to these results is that the reduction of preference reversals is an artifact of using bet pairs that do

Table 1

Classification of Descriptive Responses in Terms of Use of Probabilities and Outcomes

\begin{tabular}{|c|c|c|c|c|}
\hline \multirow[b]{2}{*}{ Verbal protocol } & \multicolumn{4}{|c|}{ Response category } \\
\hline & $\begin{array}{c}\text { Probabilities } \\
\text { only }\end{array}$ & $\begin{array}{c}\text { Amounts } \\
\text { only }\end{array}$ & $\begin{array}{c}\text { Probabilities } \\
\text { and } \\
\text { amounts }\end{array}$ & Unclassified \\
\hline $\begin{array}{l}\text { Describe briefly how you } \\
\text { chose between bets. } \\
\text { Describe briefly how you } \\
\text { came up with a price } \\
\text { for the bets. }\end{array}$ & 46 & 14 & 26 & 10 \\
\hline
\end{tabular}


Table 2

Classification of Descriptive Responses Concerning Effects of Number of Plays

\begin{tabular}{|c|c|c|c|c|c|c|}
\hline \multirow[b]{2}{*}{ Question } & \multirow[b]{2}{*}{ No } & \multirow[b]{2}{*}{ Yes } & \multicolumn{4}{|c|}{ Explanation for yes responses } \\
\hline & & & $\begin{array}{l}\text { Amount to } \\
\text { win } \\
\text { changed }\end{array}$ & $\begin{array}{l}\text { Probability } \\
\text { of winning } \\
\text { changed }\end{array}$ & $\begin{array}{l}\text { Amount } \\
\text { and } \\
\text { probabilities } \\
\text { changed }\end{array}$ & Unclassified \\
\hline $\begin{array}{l}\text { Did the number of times } \\
\text { a bet could be played } \\
\text { affect your choices? } \\
\text { Did the number of times } \\
\text { a bet could be played } \\
\text { affect the way you } \\
\text { priced the bet? }\end{array}$ & 35 & 64 & 11 & 34 & 3 & 16 \\
\hline
\end{tabular}

not conform to the usual preference reversal situation. ${ }^{3}$ In the usual situation, the distribution of the P-bet is negatively skewed with a low outcome variance, and the distribution of the \$-bet is positively skewed with a high outcome variance. However, when one considers the sampling distributions for these bets in the multiple-play situation, both differences in skewing and variance between P-bet and \$-bet are reduced. Thus, the reduction in preference reversals may be due to a reduction in the manipulated difference between the P-bets and $\$$-bets that were used.

One assumption of this interpretation is that subjects generate something like a sampling distribution of outcomes when they are presented with single bets that are represented as being played multiple times. This assumption is supported by evidence that subjects can generate and use distributional features of gambles, albeit imperfectly (we discuss this evidence in the next section). More relevant to a discussion of the preference reversal phenomenon, this interpretation assumes that higher order distributional features of the bets are crucial to defining when the preference reversal phenomenon should occur. However, past definitions of the preference reversal situation have not generally alluded to distributional features of the bets (Slovic \& Lichtenstein, 1983; Tversky et al., 1988). Instead, the preference reversal situation has been described in terms of pairing alternatives so that each has a high value on an attribute on which the other is low. Different response modes are then assumed to induce differential weighting of the attributes. The present studies contribute to this literature by suggesting that the differential weight of attributes is linked to higher order features of the outcome distributions.

\section{Multiple Plays and the Distribution of Outcomes}

Several lines of research suggest that the effects of increasing the number of plays on pricing and choice behaviors centers on how probabilities are considered and integrated with outcomes rather than on the value component of the bet alone. First, in Experiment 1, high-outcome bets produced more preference reversals than low-outcome bets for single-play conditions. This finding is inconsistent with the hypothesis that the reduction in preference reversals with more plays is due to the higher corresponding outcome values. Second,
Keren and Wagenaar (1987) also found strong effects of the number of plays on choice behavior under conditions that controlled for overall value of bets by setting outcomes levels of multiple-play bets equal to the outcomes of single bets divided by the number of plays. Because the expected values and the range of outcomes were the same for single and multiple bets in their experiment, the observed shifts in choice behavior support the hypothesis that increasing the number of plays affects how probabilities are processed. Finally, when we examine the verbal reports of subjects (Experiment 2), the most common explanation of how increasing the number of plays affected choice and pricing was that the "chances of winning" something increased with more plays.

Kahneman and Tversky (1982) argued that there are different sources of uncertainty for which different types of reasoning are relevant. Within their classification scheme, the gambles used in these and other experiments represent an external source of uncertainty for which a distributional mode of processing is most appropriate. Expected utility theory evaluates such uncertain courses of action using only the first moment (the mean) of the outcome distribution. From this perspective, it does not matter whether a choice alternative is represented as a single gamble or as a series of gambles with each outcome level divided by the number of gambles in the series, because the expected value for the two distributions will be the same. However, our experiments add to a growing body of evidence that demonstrates that subjects respond differently to single- versus multiple-play gambles in both their choice and pricing behaviors.

These differences suggest that subjects consider more than just the first moment of the distribution of outcomes when evaluating uncertain alternatives. Coombs (1975) developed a theory of risky choice that incorporates higher moments about the mean. Tests of this theory provide evidence that subjects are sensitive to shifts in variance and skewness resulting from a multiple-play representation of gambles (Coombs \& Bowen, 1971; Coombs \& Huang, 1970; Coombs \& Meyers, 1969). More recently, Lopes (1984, 1987) developed a distributional model of risk that is based on evaluating lotteries in terms of their cumulative properties. In her experimental tests (Lopes, 1984; Schneider \& Lopes, 1986), Lopes

\footnotetext{
${ }^{3}$ We are grateful to Lola Lopes for bringing this definitional argument to our attention.
} 
demonstrated how the model predicts effects of the higher moments of the distribution as well as the preference patterns for risk-seeking and risk-averse subjects. She also demonstrated how the model can incorporate differences in aspiration levels that result from differences among individuals or between task environments.

The present set of experiments suggest that the failure of expected utility theory as a descriptive theory may be due in part to the unwarranted assumption that people will use a long-run perspective when considering unique, single-instantiation events. The research reported here, and that of Keren and Wagenaar (1987), demonstrates that decision anomalies within the expected utility framework are reduced when the decision problem incorporates a long-run structure, that is, multiple instantiations of probabilistic events. Expected utility theory places this long-run frame on decision problems regardless of whether they are structured as unique or repeated events. The present results support the view that people can adopt a long-run perspective, but only when the structure of the decision task is consistent with the long-run frame.

\section{Aspiration-Level and Alternative Interpretations}

The present results are consistent with a number of different theoretical interpretations of the effects of increasing the number of plays. For example, Tversky and Bar-Hillel (1983) demonstrated that when the expected utility of a multipleplay bet is evaluated by summing the product of the multinomial probabilities of each possible outcome with the outcome's utility, a concave utility function can result in a single play of the bet having a negative expected utility (and hence not being selected) but can result in multiple plays of the same bet having a positive expected utility (and hence being selected). Thus, we can explain the behavior of Samuelson's colleague within expected utility theory using a concave utility function. Similarly, we can demonstrate that when the utility function is concave, the difference between expected utilities of the P-bet and \$-bet will decrease as the number of plays increases, thus explaining the shift of preferences toward the $\$$-bet under choice conditions. ${ }^{4}$

There are two problems with this interpretation of our experimental results. First, Samuelson's colleague and the subjects in our experiment pointed to an increased likelihood of winning (or not losing) in justifying the change in their behavior as the number of plays increased, not to a difference in the perceived outcome levels. Second, and more important, the expected utility interpretation incorporating a concave utility function cannot explain the preference reversal phenomenon in the single-play condition, nor does it describe why pricing preferences shift in the opposite direction. To do so requires additional assumptions about how pricing differs from choice. Goldstein and Einhorn's (1987) expression theory proposes that choice follows an expected utility rule using a concave utility function, but when expressing prices, subjects use a scale that is linear to dollar amounts. Although this formulation predicts preference reversals for single-play gambles and a shift in choice preference toward the $\$$-bet for multiple plays (due to the concave utility function), it does not predict a shift in the pricing preferences toward the P-bet.
To do so would require, for instance, a pricing scale that is positively accelerated on dollar amounts; however, there is little support for such a function in the literature.

An alternative interpretation of the present results focuses on how choice and pricing are made in relation to an aspiration level. The concept of aspiration level occupies an important place in theories of decision making. Simon's (1955) principle of satisficing essentially consists of the idea of setting the aspiration level at some minimum acceptable outcome level and choosing the first alternative that is likely to exceed that level. Kahneman and Tversky (1979) also incorporated the idea within prospect theory by using aspiration level as a reference point from which to evaluate gambles in terms of potential gains and losses.

The concept of aspiration level is consistent with verbal reports of our subjects (and of Samuelson's colleague) that focus on increasing the chances of winning or decreasing the chances of losing with more plays. According to this view, choice may be conceived as following a two-stage process in which the choice options that have an insufficient probability of exceeding the aspiration level are first eliminated. With increasing number of plays, the \$-bet is less likely to be eliminated during the first stage, and hence the choice shifts to some other criterion, such as potential gain.

Aspiration level can also be used to explain subjects' pricing behavior. Several researchers have suggested that subjects tend to overprice the \$-bet rather than underprice the P-bet (Lichtenstein \& Slovic, 1971; Tversky et al., 1988). For example, minimum selling prices for \$-bets often exceed their expected values. An aspiration-level interpretation of overpricing suggests that in setting a selling price, subjects may attempt to minimize the potential "loss" that occurs when the monetary gains realized by the bet exceed the selling price. Although the average "loss" for the bet in the long run will be equal to its expected value, this long-run perspective may not seem particularly relevant to the subject when the bet is played only once, because neither of the two outcomes will be equal to the expected value. For the P-bet, the amount to be won is close to the bet's expected value, but for the \$-bet, the amount to be won far exceeds its expected value. Thus, subjects may set a higher aspiration level on the \$-bet because of a greater perceived "loss," overpricing it in relation to its expected value.

When bets are represented as being played 100 times, subjects may tend to adopt the long-run perspective and evaluate the likely outcomes for both $\$$-bet and P-bet in terms

\footnotetext{
${ }^{4}$ Concavity or convexity of the utility function can produce reversals of the ordering of the expected utilities of P-bet and \$-bet with increasing number of plays. For example, given the P-bet $(.9, \$ 2)$, the $\$$-bet $(.1, \$ 100)$, and the concave utility function $u(\$)=\$^{0.5}$, a single play of the P-bet will have a higher expected utility than a single play of the $\$$-bet $(1.27>1.0)$. However, using the binomial probabilities for each of the 11 possible outcomes from playing each bet 10 times, the expected utility of the P-bet will be less than that of the \$-bet $(4.2<7.9)$. Similarly, given the P-bet $(.9, \$ 2)$, the $\$$-bet $(.1, \$ 7)$, and the convex utility function $u(\$)=\$^{2}$, the P-bet will have a lower expected utility than the \$-bet under single-play conditions $(3.6<$ 4.9), but the P-bet will have a higher expected utility when each bet is played 10 times $(327.5>93.1)$.
} 
of their long-run expectation. Aspiration levels should then approach the expected values of the bets, resulting in a reduced tendency to overprice the \$-bet. Thus, the aspirationlevel framework accounts for the pattern of results obtained in the present experiments. Finally, in a recent article, Casey (in press) used an aspiration-level framework to predict conditions under which the preference reversal phenomenon would be reversed when setting maximum bids rather than minimum selling prices. The obtained reversal of the bidchoice preference reversal when high-outcome gambles were used both supports the aspiration-level framework and calls into question the anchoring and adjustment interpretations.

\section{References}

Arrow, K. J. (1971). Essays in the theory of risk bearing. Chicago: Markham.

Casey, J. T. (in press). Reversal of the preference reversal phenomenon. Organizational Behavior and Human Decision Processes.

Coombs, H. C. (1975). Portfolio theory and the measurement of risk. In M. F. Kaplan \& S. Schwartz (Eds.), Human judgment and decision processes (pp. 63-85). Orlando, FL: Academic Press.

Coombs, H. C., \& Bowen, J. N. (1971). A test of ve-theories of risk and the effect of the central limit theorem. Acta Psychologica, 35, 15-38.

Coombs, H. C., \& Huang, L. (1970). Test of a portfolio theory of risk preference. Journal of Experimental Psychology, 85, 23-29.

Coombs, H. C., \& Meyer, D. E. (1969). Risk-preference in coin toss games. Journal of Mathematical Psychology, 6, 514-527.

Goldstein, W. M., \& Einhorn, H. J. (1987). Expression theory and the preference reversal phenomena. Psychological Review, 94, 236254.

Grether, D. M., \& Plott, C. R. (1979). Economic theory and the preference reversal phenomenon. American Economic Review, 69 , 623-638.

Kahneman, D., \& Tversky, A. (1979). Prospect theory: An analysis of decisions under risk. Econometrica, 47, 263-291.

Kahneman, D., \& Tversky, A. (1982). Variants of uncertainty. Cognition, 11, 143-157.

Keren, G., \& Wagenaar, W. A. (1987). Violation of utility theory in unique and repeated gambles. Journal of Experimental Psychology: Learning, Memory, and Cognition, 13, 387-391.

Kunreuther, H., \& Wright, G. (1979). Safety-first gambling, and the subsistence farmer. In J. A. Roumasset, J. M. Boossard, \& I. Singh (Eds.), Risk, uncertainty and agricultural development (pp. 213230). New York: Agricultural Development Council.

Lichtenstein, S., \& Slovic, P. (1971). Reversal of preferences between bids and choices in gambling decisions. Journal of Experimental Psychology, 89, 46-55.

Lichtenstein, S., \& Slovic, P. (1973). Response-induced reversal of preference in gambling: An extended replication in Las Vegas. Journal of Experimental Psychology, 101, 16-20.

Lindman, H. R. (1971). Inconsistent preference among gambles. Journal of Experimental Psychology, 89, 390-397.

Lopes, L. L. (1981). Decision making in the short run. Journal of Experimental Psychology: Human Learning and Memory, 7, 377385.

Lopes, L. L. (1983). Some thoughts on the psychological concept of risk. Journal of Experimental Psychology: Human Perception and Performance, 9, 137-144.

Lopes, L. L. (1984). Risk and distributional inequality. Journal of Experimental Psychology: Human Perception and Performance, 10 , 465-485.

Lopes, L. L. (1987). Between hope and fear: The psychology of risk. Advances in Experimental Social Psychology, 20, 255-295.

Montgomery, H., \& Adelbratt, T. (1982). Gambling decisions and information about expected value. Organizational Behavior and Human Performance, 29, 39-57.

Payne, J., Laughhunn, D. J., \& Crum, R. (1980). Translation of gambles and aspiration level effects in risky choice behavior. Management Science, 26, 1039-1060.

Pratt, J. W. (1964). Risk aversion in the small and in the large. Econometrica, 32, 122-136.

Samuelson, P. A. (1963). Risk and uncertainty: A fallacy of large numbers. Scientia, 98, 108-113.

Schneider, S. L., \& Lopes, L. L. (1986). Reflection in preferences under risk: Who and when may suggest why. Journal of Experimental Psychology: Human Perception and Performance, 12, 535548.

Siegel, S. (1957). Level of aspiration and decision making. Psychological Review, 64, 253-262.

Simon, H. A. (1955). A behavioral model of rational choice. Quarterly Journal of Economics, 69, 99-118.

Slovic, P. (1986, November). Stalking elusive preferences. Paper presented at the annual meeting of the Society for Judgment and Decision Making, New Orleans, LA.

Slovic, P., \& Lichtenstein, S. (1968). Relative importance of probabilities and payoffs in risk taking. Journal of Experimental Psychology, 78(3, Pt. 2).

Slovic, P., \& Lichtenstein, S. (1983). Preference reversals: A broader perspective. American Economic Review, 73, 596-605.

Tversky, A., \& Bar-Hillel, M. (1983). Risk: The long and the short. Journal of Experimental Psychology: Learning, Memory, and Cognition, 9, 713-717.

Tversky, A., Sattath, S., \& Slovic, P. (1988). Contingent weighting in judgment and choice. Psychological Review, 95, 371-384.

Von Neuman, J., \& Morgenstern, O. (1947). Theory of games and economic behavior. Princeton, NJ: Princeton University Press.

Received February 27, 1989

Revision received May 19, 1989 Accepted May 26, 1989 